\title{
Predicting menopausal health in a diverse population group through a theoretical linear model
}

\author{
- Theoretical model to predict menopausal health
}

\author{
Amit Sengupta $^{1,2 *}$, Nithya Srinivasan ${ }^{3}$ \\ ${ }^{1}$ Central Health Services, CGHS, Mumbai, India; \\ ${ }^{2}$ Biomedical engineering, Indian institute of technology, Delhi, India; *Corresponding Author: senguptaamit@hotmail.com; \\ ${ }^{3}$ Central Government Health Scheme (CGHS), Mumbai, India.
}

Received 24 August 2010; revised 30 August 2010; accepted 14 September 2010.

\begin{abstract}
With the increase in longevity and demographic shift, menopause is emerging as one of the major health issues affecting middle aged women in developing countries. In this study our aim was to define \& develop useful predictive indicators to assess menopausal health status in women with diverse socio-economic \& cultural backgrounds. The model was developed using the data drawn from known published works as well as our own epidemiological \& clinical case records. A linear equation was derived and expected results were obtained and analysed. The outcome was measured in terms of menopausal health \& wellbeing index. Wide cultural diversity, unequal socio-economic status and gender inequality are some of the sensitive multi factorial determinants that influence the menopausal health. Education and availability of optimal quality health care facilities positively influenced level of awareness and improved the health seeking behavior \& health literacy. The menopausal health $\&$ wellbeing index can be used as a predictive tool to develop interventional management modalities to improve quality of life.
\end{abstract}

Keywords: Menopause; Quality of Life; Urban Indian Women; Socio-Cultural Factors; Health Literacy; Theoretical Model

\section{INTRODUCTION}

Women constitute $70 \%$ of world's poorest and $90 \%$ of the landless people. In India, $60 \%$ of women beyond the age of 50 are widows, and more than $80 \%$ elderly women beyond 60 years of age live in extended families with grown up children, yet more than $80 \%$ suffers affective syndrome [1,2]. With rapid demographic changes, the number of elderly population is gradually rising and the ratio of elderly \& young adults is expected to reach $1: 4$; and by 2020 , the actual number of women aged more than 50 will be nearing 150 million in India. The situation in the developed countries is more alarming with the ratio reaching $1: 2$, showing a high dependency rate. Thus, menopausal health assumes greater significance especially for those who suffer symptom and substantial morbidity. Moreover, menopausal health which signifies overall health and well being status of a woman during and beyond middle age is also linked to various socioeconomic, cultural, physiological as well as psychological factors. Though physiological alteration in the cyclical ovarian function begins early at the commencement of climacteric period i.e., around 35 years of age but morbid conditions such as vasomotor instability, psychosexual problems, mental-physical exhaustion, anxiety-palpitation or genitourinary discomfort, bony pains in general and osteoporosis, diabetes \& coronary arterial disease in particular are commonly seen later during and/or after menopause. They are generally ignored in rural, sub-urban as well as urban India, even if they suffer from symptoms silently [1-6]. However, menopause is gradually emerging as an issue in India where traditionally it was linked to aging. M. Flint [4] and M. Bourlet [7] did extensive Socio-anthropological and morbidity studies on menopause in India and South East Asian countries. Scarce availability of quality health care facilities, gender inequalities, lack of awareness in rural, semi-urban and urban areas prevents women from seeking much needed medical support for conditions such as osteoporosis, recurrent GUI (genitourinary infections), SUI (urinary stress incontinence), DM (Diabetes mellitus), HT (Hypertension), CAD (coronary arterial disease) and affective disorders etc. The lack of assured basic 
health care facilities, poverty, malnutrition, illiteracy was considered some of the reasons for imperceptible improvement in their health in poor developing countries $[8,9]$. Even in US low income and poor education was found to be associated with increased menopausal symptoms and determining age of peri menopause [10, 11]. Income, place of residence (urban/rural) and education level of women also modifies health seeking behavior and house hold expenditure on health $[8,9]$. Studies also suggested a link between early life factors, good health care support received during the reproductive period and better health status of women entering menopause or the phase of reproductive agin [12]. It is also well known that the women across different cultures and ethnicity perceive and behave differently during menopause. The cultural attributes affect women's menopausal experience in a very complex way $[1,3,4,7,13-18]$. Traditionally India is a multilingual, multi ethnic, multireligious nation where population co-exists concurrently in societies corresponding to ancient, medieval and modern era, and as a result wide variations in their health status and human development index is seen. Apparent positive mental attitude towards aging and/or menopause is attributed to the cultural upbringing whereas on the contrary it also points toward their negative health seeking behavior [1]. Great efforts have been made by developed nations to alleviate sufferings through a multidisciplinary approach where symptoms expressed during menopause have been quantified in the form of different menopause rating scales [19-21]. But escalating cost and recent controversies surrounding hormone therapy appeared to have raised some concerns on the issue of quality of life of women entering menopause-climacteric. Thus, a complete review of issues and factors such as demographic, nutritional, life style linked to menopause and its management is currently underway in the developed world [22,23]. In India where it was poorly studied, acquiring \& generating population based specific information on menopause is of vital interest to the policy makers. The task is not only difficult but also quite challenging because of non-homogeneous diversified nature of population group and many contradictions, whereby rating health related quality of life appeared difficult; some of them is gross inequitable distribution of wealth and gender gap which affect women's morbidity pattern and health seeking behavior differently. Thus, in any such given situation where it is difficult to define or predict complex biological or epidemiological event, modeling is a useful alternative tool, which when properly constructed by simplifying various complex assumptions, may predict the outcome closer to real clinical studies. We proposed a theoretical model to assess \& predict inter-relationship between key human development indices, behavior, attitude and morbidity pattern during menopause and expect the model to be useful in suggesting preventive \& interventional modalities that can be incorporated in the health care delivery management protocol so as to prevent menopause related morbidities.

\section{METHODS AND MATERIALS}

Menopause is influenced by socio-economic, gender, cultural, behavioural, biological, physical and endocrinal factors therefore an integrated multi-factorial model on menopausal health in any diverse heterogeneous population group will be an appropriate tool. In order to construct a model, relevant data for India were acquired from the published research papers $[1,2,5,6,24,25]$. In addition, the model was also validated with the support of some interesting data generated from retrospective analysis of the records of 209 middle aged women belonging to an economically privileged group which was initially not intended for research purpose but basically compiled for our routine office purpose. Written and verbal consent to fill up the pre-treatment questioner are routinely taken from every woman attending our menopause clinic as part of the clinical protocol. Based on analysis of the data and keeping in view some of the key causative attributes linked to menopause, a simple linear model was proposed and validated. Menopausal health $\&$ wellbeing constant was derived.

\subsection{Formulation of a Theoretical Model}

1) Quantification of menopausal health \& wellbeing of a woman requires formulation of an index or measuring scale. India and Asia have pluralism and heterodoxy where it is difficult to acquire specific data for quantification or standardization of the quality of life index. In the absence of specific real time data, theoretical model is an ideal \& powerful decision making tool that can solve various complexities linked to biological or human phenomenon. However, real complex assumptions need to be simplified in order to construct a model and apply it to predict the outcome from an observational study. The model should be validated using patient/population specific data from the studies. We studied socio-economic data, morbidity pattern, relevant investigation and treatment follow up details from both published as well as unpublished sources as mentioned above. In this study, broadly five relevant parameters were considered based on our search findings (multi factorial model). They are as follows: For socio-economic status, we have considered UNDP's Human development index (HDI) [2,24,25] that included most of the relevant factors enumerated 
below e.g., the socio-economic security, education access, life skill development, life expectancy, literacy rate, the women's rights issues like gender inequality i.e., given by Gender development index (GDI) inequalities . It is simply the HDI adjusted downwards for gender inequalities. HDI - GDI = measures gender disparities; GE (Gender equality) is good if HDI - GDI (penalties for gender inequalities) is low and poor if HDI - GDI is high. GDI measures achievements in the same dimensions and variables as the HDI but captures inequalities and is uniformly low (penalty for gender inequalities) for most of the developed nations with homogeneous population groups [24]. For our model we added the actual figures for HDI \& GDI to observe overall combined impact both the indices (Development indices - DI) may have on menopause.

2) Awareness on menopause \& all its aspects (Menopausal health literacy-MHL): Percentage of women aware of menopause has been calculated as median value varying between 0 (none of the women aware of menopause) to 1 (All women aware of menopause).

3) Attitude/belief towards menopause (Ac) is linked to their cultural attributes. It is the percentage of women who believe menopause to be a positive event in their life cycle. The calculated median value varies between 0 (none with positive attitude) to 1 (all with positive attitude).

4) Morbid symptoms (Mo) related to menopause was either expressed by women or retrieved by the physicians. Median value varies between 0 (none with symptoms) to 1.0 (all with symptoms).

\subsection{The Equation}

It is given by a simple linear mathematical arithmetic equation as proportionality constant $(\delta \kappa)$ for menopausal health. This was to observe the multi factorial effects that may exert its influence in determining menopausal health of women.

MHI $\alpha$ DI (menopause health index directly proportional to development index)

MHI $\alpha$ MHL (MHI directly proportional to menopause health literacy)

MHI $\alpha$ Ac (MHI directly proportional to attitude/culture/belief)

MHI $\alpha$ 1/Mo (morbidity), i.e., short term and long term morbidity

Whereas SCE (Socio-Cultural-Educational-Economic attributes $)=(\mathrm{DI}+\mathrm{MHL}+\mathrm{Ac})$

Therefore, from (a) \& (b), we derived MHI $=\delta \kappa(\mathrm{SCE})$ / Mo for a particular time period (t0....tn)

While assuming $\delta \kappa=1$ (non-zero) menopausal health proportionality constant for all cohort group.
Based upon our observation, we expect a linear relationship between reductions in morbidity and SCE, thus when we plot a graph between Mo Vs SCE, we may get a descending curve. An ascending curve is derived when we plot MHI Vs SCE, i.e., with improvement in SCE the $\mathrm{MHI}$ also improves and vice versa, assuming the value for Mo to fall. While computing data from longitudinal prospective survey to observe the effect over a period of time in a community, the constant fall and rise in the value of $\delta \kappa(t 0, t 1 \ldots . t n)$ would suggest gradual improvement and deterioration respectively over a period of time in menopausal health. The value $\delta \kappa$ will approach unity (1.0) when the morbidity becomes negligible theoretically but for all practical purposes it is not achievable. Given the unit value of $0-1$ (median value) for each variable, the calculated MHI value will swing between the range of $[(0.0)$ to $(\alpha)$ (most unfavorable to most favorable)] theoretically, whereas actual values will be fluctuating in a range bound manner as shown below (Eqs.1-3).

\subsection{Validation \& Data Retrieval}

Details such as age, age at menopause, duration of menopause, gross family income, level of education, present/past medical history, personal history, cultural practices, belief, attitude, awareness on menopause/HRT, perceived relief, source of anxiety regarding HRT, etc, were included. A number of western studies are available to study HRQoLI (health related quality of life index) during menopause. The questionnaire format that is followed in our menopause clinic on quality of life indices and morbidity patterns were developed following extensive search of literature to ensure its validity and reliability [2,19-21]. As part of the protocol, routine and specialized tests such as blood sugar, CBC, lipid profiles, ECG, pap screening, complete biochemical, endocrinal, radiological and color Doppler-sonography tests, besides dual energy bone densitometry and sono-mammography were done as and when required. Their height, weight, BMI, blood pressure recorded and other relevant systemic, breast and gynaecological/pelvic examination including PAP test were also done routinely. The statistical analysis was carried out using SPSS-PC program (version 11.0 for windows). A descriptive analysis was performed for population variables as mentioned above and binominal distribution, chi-square and log regression tests were used to study qualitative variables.

\section{RESULTS}

We observed that the MHI (menopause health index) calculated from the derived equation can be used for conducting comparative studies between rural, semi-urban 
and urban population. In the absence of well established HRQLI (health related quality of life index) for a nonhomogenous population group belonging to developing countries such as India. We arrived at some figures for MHI as shown below for the study purpose. A marked difference was observed between urban metro economically privileged population (our group) with MHI of 4.8 and rural/semi-urban population with MHI of 2.6. (Data from other sources are compared, see the text above). (Eqs.1,2), assuming the menopausal constant $(\delta \kappa)$ as 1 . India $($ overall $)=0.5+0.5+0.3+0.8 / 0.8=2.6($ low $\mathrm{MHI})$

India (metro-middle class) $=0.8+0.8+0.7+0.6 / 0.6$

$=4.8$ (Middle $\mathrm{MHI}$ )

(HDI \& GDI $=0.5 \& 0.8$ for an average Indian \& metro middle class Indians respectively; assuming an approximate value for calculation purpose, the median value for knowledge of menopause amongst rural and urban population is 0.3 and 0.7 respectively; median value for positive belief or attitude amongst rural \& urban population is given the value of 0.8 and 0.6 respectively; average/median value of women expressing/ or retrieved or suffered from menopause symptoms in rural \& urban population was 0.6 .

For developed countries where GDI \& HDI is constant for a homogeneous population groups, the comparison was done on the basis of known specific menopause morbidity scales such as Greene [19], MRS [20] etc., The other two parameters i.e., awareness level and attitude was also taken into consideration [11,13,14,19$21,24,25,28]$. For example, if we compare the two groups of population e.g., Singapore (ASEAN) or Tokyo (Japan) or and Sweden (Europe-west), they have more or less a similar development index; HDI, GDI \& awareness level assumed to be constant but their difference can be described on the basis of their different cultural attitude and perception of symptoms as well as actual short term \& long term morbidity.

For developed nations or for a small group of affluent Indian urban women, it could be $=0.9+0.9+0.9+0.7$ / $0.6=5.6$ (high $\mathrm{MHI})$

\section{DISCUSSIONS}

The discussion focuses on validating the derived equations taking into consideration data retrieved from literature as well as our own case records wherever applicable. The values for the cohort/country etc. are derived from UNDP reports and various other studies as mentioned in the reference section. For India which is a developing country with non-homogeneous population, comparative values for cohort groups e.g., rural, semiurban or urban-metro cities have been derived using data from the present study as well as from the published work [1,2,5,6,8,9,16,24-27]. The general profile of our economically privileged \& medically insured middle aged urban (METROPOLITAN) women showed a very high literacy rate of $93 \%$ with $36 \%$ university graduates as compared to the national female literacy rate of 54\% (urban vs. rural :73 vs. 46 ). $43 \%$ of them were employed in organized sectors as against the national average of $30 \%$ who are economically active, mostly doing menial jobs in unorganized sectors. Amongst economically privileged urban population, $86 \%$ and $65 \%$ women with graduate educational qualification were aware of menopause and HRT respectively. The ODD'S of not knowing menopause as a clinical entity and HRT as therapeutic modality was $4.5(\mathrm{p}<0.005)$ and $3.4(\mathrm{p}<0.01)$ times greater in women with secondary level education (high school completion) as compared to the university graduates. The ODD'S of not being able to express or link hot flushes with menopause was $2.2(\mathrm{p}<0.07)$ times greater in women who had no knowledge of menopause. The mean age of menopause in our study group was 45yrs, surgical $(43+\ldots$ byrs $)$ : natural $(48+\ldots 4 y r s)$. Women belonging to underprivileged group's experienced early menopause i.e., mean age vary from 40 to 48 years [6,26-28] The mean age of menopause in developed countries is higher and varies between 48-51 years [17]. Our economically privileged educated urban women appeared less positive $(47.6 \%)$ when compared with the economically deprived rural \& semi-urban Indian women (94\%). $88.83 \%$ of all women from our urban metro group consulted physicians practicing evidence based medicine. Only $8 \%$ women with the knowledge of menopause do not express any morbid symptoms related to peri-menopause compared to $20 \%$ \& $35 \%$ who had vague knowledge or no knowledge about menopause during peri-menopause period respectively. Menstrual irregularity $(32 / 83=38.5 \%)$, psychosomatic problems $(17 / 83=20.5 \%)$ and the bony or musculoskeletal pains $(10 / 83=12 \%)$ were three most common morbid conditions affecting the quality of life in our economically privileged urban women during peri-menopause. Genitourinary morbidity was more beyond 65 yrs of age. The educated urban women reported more vasomotor symptoms-VMS (71-86\%) as compared to $17.1 \%$ in semi urban India. Better awareness level of educated \& economically privileged urban women appears to be an influencing factor in expressing the morbid symptoms thereby seeking early remedial measures compared to those who were generally unaware of menopause and suffers silently. Nearly one-quarter of Japanese community-dwelling, healthy women in the peri- and post menopausal states suffered from menopausal symptoms, which decreased their quality of life in everyday life [29]. The Chinese women displayed a low to moderate fre- 
quency of reporting symptoms across vasomotor, vaginal, sleep-related, cognitive, emotional and somatic categories. Their attitude toward menopause and aging tended to be more positive, neutral or ambivalent, as opposed to negative [30]. The affluence of our emerging middle class had a direct influence on BMI, majority of them were found to be overweight. Obesity or high BMI $(>25)$ was found in $59 \%$ women in our group as compared to the national average of $11.6 \%$. BMI and morbidity: A link was found between overweight or obesity (high BMI) and psychosomatic illness, hypertension, diabetes \& alcohol intake. The absence of anemia also suggested good nutritional status amongst our affluent group. In India $60 \%$ of all women between 15 to 60 years of age are anemic. [2,27]. Alcohol and Smoking: All women consuming alcohol in our study group were overweight and on HT/HRT therapy. $70 \%$ had surgical menopause. $25 \%$ of women smokers in the group were on HT/HRT and 50\% had normal menopause. Natural and Surgical Menopause: More women suffered hot flushes following the hysterectomy compared to those who had natural menopause [(55/67 (82\%) vs. 75/110(68\%) p < 0.05)]. We also observed higher hysterectomy rate (14\%) amongst the urban educated women attending our menopause clinic compared to $5.8 \%$ in semi urban India [5]. $28.9 \%$ of women were individualized to receive different types of hormone therapies including Soya - isoflavones for various menopause related morbidity at any given point of time in our group. This was in sharp contrast to almost none from the semi urban areas receiving HT/HRT even though they silently suffered symptoms almost equally [5]. In one of the recent studies, $58 \%$ of African-American women used HRT or considered using it [31]. The use of most types of CAM were not related to menopausal status or symptom reporting but to socio-demographic factors, co-morbidities, and health behaviours as reported in another US study [32]. Based upon the analysis of the data as mentioned above, we defined certain broad intervention modalities i.e., firstly, to promote positive attitude, secondly, to improve the developmental indices i.e., HDI \& GDI and thirdly to reduce the severity of menopausal symptoms Attitude can be improved through positive thinking, and integrating complimentary therapies like yoga and counseling and also multi-cultural integration of various culturally secluded groups towards a common goal of healthy living; it was observed that as long as the Indian women remained within their cultural sphere, they did not show much appreciable behavioral changes and suffered from mental or bodily symptoms silently without being aware of the need for intervention. Mental health can also be improved through promotion and preservation of extended family support systems. We also must promote life style change through exercises, regulating the diet in order to maintain the ideal body weight and reduce menopause related diseases. Women should be educated \& economically empowered which in turn will help in generating awareness on menopause \& its short long term health related changes. Attention should also be focused on the availability of various therapeutic modalities. Efficient evidence based health care facilities should be strengthened and extensive studies be initiated to assess the menopause related morbidity across various cross sections of population. Medical audit is necessary to reduce surgical (total hysterectomy) interventions for DUB. Overall objective behind doing this is to improve quality of life. We felt while general family physicians may assume the key role in conducting primary health screening, high risk severe cases may be referred to a specialist for better management of specific conditions such as severe psycho-motor symptoms, osteoporosis, stress urinary incontinence etc. after assessing them using known scoring system e.g., MRS, Greene etc.

Summarizing:

EDUCATION-improves body-mind interaction (Knowledge/awareness/Attitude/Belief)

ECONOMY-improves Nutrition \& self confidence but overweight becomes a problem,

AVAILABILITY of service-improves Health seeking behavior \& expression

AUDIT-Promotes evidence based medicine (EBM) vis-à-vis cultural practices, which will be helpful in analyzing use of various modalities of treatment, evidence based or traditional. Unless corrective measures are taken now, global economy will be seriously affected due to ever growing number of elderly women suffering from symptoms and morbidity.

\section{CONCLUSION}

An integrated MHI is useful for comparative analysis as well to observe the gradual improvement in menopausal health status of middle aged women in a developing country. Based on the equation and the derived values, we conclude that higher level of education \& income, greater awareness positively influenced the quality of health of middle aged women during menopause. Health seeking behavior of women, attitude of health care providers and availability of quality health care facilities may affect treatment and management modalities during menopause. In general, there is a greater need to make women and society in a developing country health literate i.e., providing them with the right kind of information about menopause, suggesting alternatives to hysterectomy and importance of individualized hormone replacement therapies or modulators or Soya (isoflavones) 
to alleviate severe acute symptoms and long term problems like osteoporosis, Alzheimer's etc. Greater emphasis should be laid on regular health check up of middle aged women in order to identify the impending risks of hypertension, diabetes mellitus, coronary arterial diseases etc.

\section{AUTHOR'S INFORMATION}

ASG is the consultant in obstetrics and gynecology as well as a professor of biomedical engineering in a premier teaching and research institute, working towards betterment of women through use of innovative biomedical technology and analysis. NS is a research worker currently working in the field of cancer cell biology.

\section{ACKNOWLEDGEMENTS}

The authors show their sincere gratitude to Professor Prakasam, Professor of Biostatistics, International Institute of Population studies, Mumbai for making facilities available at the data centre and for his technical help \& constructive inputs in analyzing the data. The authors would also like to thank sincerely Dr. Prasad Bonerjee, Research scholar at IIPS, Mumbai, for formatting the pre-tested structured menopause questioner forms into a SPSS compatible data sheet, and for training us in using SPSS system while solving problems in data handling. We specially acknowledge the helpful discussions we had with Dr. Sainani, Additional Director of CGHS Mumbai on epidemiology \& women issues. We would also like to thank Ms. Sonia Dharware, staff nurse, who meticulously maintained and preserved all the case records.

Conflict of interest-None

Source of funding-None

\section{REFERENCES}

[1] Sengupta, A. (2003) The emergence of the menopause in India. Climacteric, 6, 92-95.

[2] WHO (2000) Women of South East Asia, a health profile. New Delhi: Regional publications SEARO, 34.

[3] Bush, T.L. (1990) The epidemiology of cardiovascular disease in post menopausal women. Annals of the New York Academy of Sciences, 592, 263-271.

[4] Flint, M. and Samil, R.S. (1990) Cultural and sub-cultural meaning of the menopause. Annals of the New York Academy of Sciences, 592, 134-148.

[5] Kaur, S., Walia, I. and Singh, A. (2004) How menopause affects the lives of women in suburban Chandigarh, India. Climacteric, 7(2), 175-180.

[6] Singh, A. and Arora, A.K. (2005) Profile of menopausal women in rural north India. Climacteric, 8(2), 177-184.

[7] Boulet, M.J., Oddens, B.J., Lehert, P., Vermer, H.M. and Visser, A. (1994) Climacteric and menopause in seven SE Asian countries. Maturitas, 19, 157-176.

[8] The World Bank (1996) Improving women's health in India. Development in Practice Series, New York, Oxford University press for the WB.
[9] Rout, H.S. (2006) Linkages between income education and health: Case of rural Orissa. The Indian Economic Journal, Indian Economic Conference Volume, 70-79.

[10] Wise, L.A., Krieger, N., Zierler, S. and Harlow, B.L. (2002) Lifetime socioeconomic position in relation to onset of per menopause. Journal of Epidemiology and Community Health, 56, 851-860.

[11] Brzyski, R.G., Medrano, M.A., Hyatt-Santos, J.M. and Ross, J.S. (2001) Quality of life in low-income menopausal women attending primary care clinics. Fertility and Sterility, 76(1), 44-50.

[12] Mishra, G.D., Cooper, R., Tom, S.E. and Diana, Kuh. (2009) Early life circumstances and their impact on menarche and menopause. Women's Health, 5(2), 175-190.

[13] Parsons, M.A. and Obermeyer, C.M. (2007) Women's midlife health across ultures: DAMES comparative analysis. Menopause, 14(4), 760-768.

[14] Robin, G. and Nanette, S. (2009) Menopausal symptoms and ethnicity: The Study of women's Health Across the Nation. Women's Health, 5(2), 127-133.

[15] Christopher, E. and Jacqueline, S. (2003) Reproductive and sexual health of older women in developing countries. British Medical Journal, 327, 64-65.

[16] Gupta, P., Sturdee, D.W. and Hunter, M.S. (2006) Midage health in women from the Indian subcontinent (MAHWIS): General health and the experience of menopause in women. Climacteric, 9(1), 13-22.

[17] Sommer, B., Avis, N., Meyer, P., Ory, M., Madden, T., Kagawa-Singer, M., Mouton, C., Rasor, N.O. and Adler, S. (1999) Attitudes toward menopause and aging across ethnic/racial groups. Psychosomatic Medicine, 61(6), 868875.

[18] Loutfy, I., Abdel Aziz, F., Dabbous, N.I. and Hassan, M.H. (2006) Women's perception and experience of menopause: A community-based study in Alexandria, Egypt. Eastern Mediterranean Health Journal, 12(Suppl 2), S93106.

[19] Greene, J.G. (1998) Constructing a standard climacteric scale. Maturitas, 29(1), 25-31.

[20] Schneider, H.P.G., Heinemann, L.A.G. and Thiele, K. (2002) Menopause rating scale (MRS): Linguistic and cultural translation into English. Life and Medical Science Online, 3, 1-13.

[21] Zollner, Y.F., Acquadro, C. and Schaefer, M. (2005) Literature review of instruments to assess health-related quality of life during and after menopause. Quality of $\mathrm{Li}$ fe Research, 14(2), 309-327.

[22] Gold, E.B., Block, G., Crawford, S., Lachance, L., FitzGerald, G., Miracle, H. and Sherman, S. (2004) Lifestyle and Demographic Factors in Relation to Vasomotor Symptoms: Baseline Results from the Study of Women's Health Across the Nation. American Journal of Epidemiology, 159, 1189-1199.

[23] Melby, M.K., Lock, M. and Kaufert, P. (2005) Culture and symptom reporting at menopause. Human Reproduction Update, 11(5), 495-512.

[24] Bardhan, K. and Klasen, S. (1999) UNDP Gender Related indices: A critical review. World Development Report, 27, 985-1010.

[25] United Nations Development Programme (1999) Human development report. Oxford University Press, New York.

[26] Syamala, T.S. and Sivakami, M. (2005) Menopause, an 
emerging issue in India. Economic and Political Weekly, 40, 4923-4930.

[27] National Family Health Survey (NFHS-2), 1998-99, under Reproductive and child health project. International Institute for Population Studies (2001, phase I\&II), Mumbai, India.

[28] Gold, E.B., Bromberger, J., Crawford, S., Samuels, S., Greendale, G.A., Harlow, S.D. and Skurnick, J. (2001) Factors associated with age at natural menopause in a multiethnic sample of midlife women. American Journal of Epidemiology, 153(9), 865-874.

[29] Satoh, T. and Ohashi, K. (2005) Quality-of-life assessment in community-dwelling, middle-aged, healthy women in Japan. Climacteric, 8(2), 146-153.

[30] Shea, J.L. (2006) Chinese women's symptoms: Relation to menopause, age and related attitudes. Climacteric, 9 (1), 30-39.

[31] Sharps, P.W., Phillips, J., Oguntimalide, L., Saling, J. and
Yun, S. (2003) Knowledge, attitudes, perceptions and practices of African-American women toward menopausal health. Journal of National Black Nurses' Association, 14(2), 9-15.

[32] Gold, E.B., Stemfield, B., Kelsey, J.L., et al. (2000) Relation of demographic \& life style factors to symptoms in a multiracial/ethnic population of women 40-55 years of age. American Journal of Epidemiology, 152, 463-473.

[33] Cristina, K., Haider, M.A., Junior, J.M.S., Nunes, M.G., Quadros, L.G.A., Kemp, C., et al. (2006) Randomized clinical trial comparing CEE and isoflavones in post menopausal women-apilot study. Maturitas, 53, 49-58.

[34] Kronenberg, F. (1990) Hot flushes: Epidemiology \& physiology. Annals of the New York Academy of Sciences, 592, 52-86.

[35] Thompson, B., Hart, S.A. and Dumo, D. (1973) Menopause age and symptomatology in a general practice. Journal of Biosocial Science, 5, 71-82. 